\section{HVEM protects mucosa}

Herpes virus entry mediator (HVEM) is a member of the family of tumor-necrosis factor (TNF) receptors and is reported to be a risk trait for colitis. In Nature, Kronenberg and colleagues show that HVEM expressed on gut and lung epithelial cells contributes to innate mucosal responses. After infection, ligation of HVEM with the costimulator CD160 expressed on intraepithelial lymphocytes and $\gamma \delta$ T cells elicits the production of antimicrobial peptides, interleukin 6 (IL-6) and TNF. Blockade of CD160, but not of the other HVEM counter-ligands BTLA or Light, blunts this response in vivo. HVEM signaling stabilizes the kinase NIK, which in this pathway activates the transcription factor STAT3 in an as-yetunknown way. HVEM and IL-22 act in synergy to protect mucosal tissues, as genetic ablation of either renders mutant mice more sensitive to citrobacter or streptococcal infection. How the HVEM-CD160 interaction is regulated in quiescent tissues and is activated after loss of mucosal integrity should be a focus of future studies.

Nature 488, 222-225 (2012)

\section{Bridging inflammation in obesity}

Obesity is associated with a distint inflammatory phenotype in which free fatty acids (FFAs) such as palmitate can elicit the inflammatory cytokines TNF and IL-6 from adipose tissue. In Nature Medicine, Bhattacharya and colleagues demonstrate a key intermediary role for fetuin A (FetA) in obesity-dependent inflammation. FetA is a glycoprotein secreted by the liver and is an important transporter of FFAs. The authors find more FetA in obese patients and show that in vivo knockdown of either FetA or TLR4 diminishes the inflammatory signature in two different experimental models of mouse obesity. FFAs do not bind TLR4 directly but instead do so via FetA. Indeed, the authors confirm FetA-TLR4 binding by both immunoprecipitation and surface plasmon resonance. A complex of FetA and FFA is required for optimal activation of transcrition factor NF- $\mathrm{KB}$ proinflammatory cytokine signaling. The output of FetA by the liver therefore has an fundamental influence on the inflammation triggered by FFAs.

Nat. Med. 18, 1279-1285 (2012)

\section{Circadian rhythms}

Leukocyte numbers in the blood and release of hematopoietic progenitor cells from the bone marrow are known to undergo circadian oscillations. In Immunity, Frenette and colleagues show that the recruitment of leukocytes to tissues in the steady state also has circadian rhythms orchestrated by sympathetic nerves. The rhythmic recruitment of leukocytes to the bone marrow and skeletal muscles peaks at night and correlates with fluctuation in the expression of adhesion mediators (ICAM-1 and CCL2) on epithelial cells in muscle or of rolling and adhesion mediators (selectins and VCAM-1) in the bone marrow. Oscillations in the expression of selectins and integrin receptors are induced through local adrenaline signaling via $\beta$-adrenoreceptors and are ablated by changes in the light cycle. Because humoral factors are also known to regulate circadian rhythms, neural and hormonal pathways probably act together to fine tune these responses. Immunity 37, 290-301 (2012)

\section{Pathogens and commensals}

Whether tolerance to commensals is lost during gastrointestinal infection is unclear. In Science, Belkaid and colleagues use a model of infection with Toxoplasma gondii to assess the effector and memory $\mathrm{T}$ cell responses generated against commensals during the course of a primary immune response to a pathogen. During infection with $T$. gondii, the ignorance of $\mathrm{CD} 4^{+} \mathrm{T}$ cells to commensal antigens is lost and microbiotaspecific $\mathrm{T}$ cells are activated and differentiate into T helper type 1 cells but not into cells of the $\mathrm{T}_{\mathrm{H}} 17$ subset of helper $\mathrm{T}$ cells or regulatory $\mathrm{T}$ cells. Commensal-specific $\mathrm{T}$ cells activated during infection survive long term and persist in extralymphoid tissue in a manner similar to that of pathogen-specific memory cells, and a fraction of them become activated during secondary infection. As bacteria colonize not just the gut but also the skin and the lung, immunity at these barrier sites can be influenced by responses to commensals as well.

Science (23 August 2012) doi:10.1126/science.1220961

\section{MicroRNA-based resistance to malaria}

Erythrocytes heterozygous (HbSA) or homozygous (HbSS) for the gene linked to sickle cell anemia have well-documented resistance to infection by the malaria parasite Plasmodium falciparum. In Cell Host \& Microbe, Chi and colleagues describe a radical, previously unknown mechanism of sickle cell-dependent resistance to $P$. falciparum. Although they lack a nucleus, erythrocytes are replete with microRNA. The authors find that HbSA erythrocytes and, even more notably, HbSS erythrocytes are especially enriched for four microRNAs (Let7i, miR181, miR223 and miR451) and that these are actively translocated to the parasite. Through the use of transfection and antagomirs, the authors demonstrate that miR451 is especially important in controlling parasitemia. As orthologous microRNA-processing machinery is absent from $P$. falciparum, miR451 seems to abrogate parasite translation by a previously unknown mechanism. This microRNA covalently binds to a specific parasite mRNA (PKA-R) to form a single contiguous mRNA molecule that is poorly translated. As PKA-R is important for $P$. falciparum growth, this represents a potentially important endogenous mechanism of regulating parasite growth.

Cell Host Microbe 12, 187-199 (2012)

\section{Nuclear silencing}

Immunoglobulin genes are known to localize to distinct nuclear compartments during B lineage development and reposition when variable-diversity-joining recombination is occurring. Association with the nuclear lamina silences gene transcription and precludes gene rearrangements. In Cell, Zullo et al. identify GAGA-rich lamina-associated sequences in the immunoglobulin heavy-chain locus (Igh). The entire Igh locus associates with lamin B in fibroblasts. The transcription factor ThPOK, in complex with the histone deacetylase HDAC3 and lamin protein Lap $2 \beta$, promotes interaction of lamina-associated sequences with the nuclear lamina. Treatment with HDAC inhibitors or knockdown of cKrox or HDAC3 releases Igh; however, reassociation with the nuclear lamina requires passage through mitotic anaphase. How these interactions are developmentally regulated during B cell differentiation remains unknown.

$L A D$ 